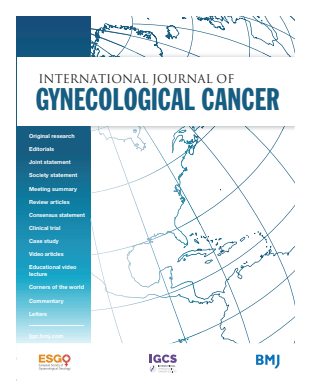

\title{
Postoperative complications of epidural analgesia at hysterectomy for gynecologic malignancies: an analysis of the National Surgical Quality Improvement Program
}

Sarah A Ackroyd (D) , ${ }^{1}$ Enrique Hernandez, ${ }^{1,2}$ Maureen E Roberts, ${ }^{1,2}$ Christina Chu, ${ }^{1,2}$ Stephen Rubin, ${ }^{1,2}$ Gina Mantia-Smaldone, ${ }^{1,2}$ Karen Houck ${ }^{1,2}$

${ }^{1}$ Obstetrics, Gynecology, and Reproductive Sciences, Temple University Hospital, Philadelphia, Pennsylvania, USA

${ }^{2}$ Division of Gynecologic Oncology, Fox Chase Cancer Center, Philadelphia, Pennsylvania, USA

\section{Correspondence to} Dr Sarah A Ackroyd, Obstetrics, Gynecology, and Reproductive Sciences, Temple University Hospital, Philadelphia, PA 19140-5192, USA; sackroyd15@gmail.com

Society of Gynecologic Oncology 50th Anniversary Annual Meeting on Women's Cancer. March 2019, Honolulu, HI.

Received 26 February 2020 Revised 28 April 2020 Accepted 30 April 2020 Published Online First 25 May 2020

D Check for updates

(c) IGCS and ESGO 2020. No commercial re-use. See rights and permissions. Published by BMJ.

\begin{tabular}{l}
\hline To cite: Ackroyd SA, \\
Hernandez E, Roberts ME, \\
et al. Int J Gynecol Cancer \\
2020;30:1203-1209. \\
\hline
\end{tabular}

\section{HIGHLIGHTS}

- Large database study including more than 2035 patients receiving epidural with hysterectomy for malignancy.

- Epidural was associated with higher complication rates, but mortality was unaffected.

- Epidural was associated with longer length of stay and more readmissions.
(10.1\% vs $7.2 \%)$; and delay in return of bowel function (12.3\% vs $9.3 \%$ ) (all $\mathrm{P}<0.05)$. Hospital length of stay was significantly longer in the epidural group as compared with the no-epidural group (5.69 days vs 4.79 days, $P<0.01$ ) and readmissions were higher in the epidural group (10.5\% vs $9.7 \%, P<0.01)$, but there was no difference in 30-day mortality between the groups ( $\mathrm{P}=0.62)$.

Discussion The rate of 30-day complications and length of stay among women undergoing an abdominal hysterectomy for gynecologic malignancy was higher for those who received epidural analgesia, but there was no difference in 30-day mortality. Although epidural analgesia can provide a number of benefits when used for postoperative pain control, the possible association with increased 30-day morbidity and length of stay needs to be considered.

\section{INTRODUCTION}

Regional anesthesia provided through an epidural catheter is an effective analgesic method to improve pain control and minimize undesirable side effects of systemic opioid medications. Studies of epidural use in abdominal hysterectomy for benign disease have demonstrated lower mean pain scores, decreased morphine use, shorter time to return of bowel function, and higher patient satisfaction when compared with patients who received oral and intravenous opioid-based care. ${ }^{12}$

Though studies demonstrate benefit in women undergoing gynecologic surgery for benign disease, studies in gynecologic oncology patients have been less consistent. While some have reported improved pain scores and opioid-sparing effects, ${ }^{34}$ others have noted no difference in pain control or opioid use. ${ }^{5}$ Despite variation in efficacy of reported pain control, multiple studies demonstrate mixed reviews on post-operative complications with use of an epidural. ${ }^{3-6}$ Epidural use has been found to 
Table 1 Patient characteristics

\begin{tabular}{|c|c|c|c|c|c|c|}
\hline \multirow[b]{2}{*}{ Variable (\#, \%), (mean+SD) } & \multicolumn{3}{|l|}{ Unmatched } & \multicolumn{3}{|c|}{ Propensity-matched } \\
\hline & $\begin{array}{l}\text { No-epidural } \\
n=14157\end{array}$ & $\begin{array}{l}\text { Epidural } \\
\mathrm{n}=2035\end{array}$ & P-value & $\begin{array}{l}\text { No-epidural } \\
\mathrm{n}=\mathbf{2 0 3 5}\end{array}$ & $\begin{array}{l}\text { Epidural } \\
\mathrm{n}=2035\end{array}$ & SMD \\
\hline Ascites & 1505 (10.6) & $279(13.7)$ & $<0.001$ & $285(14.0)$ & $279(13.7)$ & 0.011 \\
\hline \multicolumn{7}{|l|}{ ASA class } \\
\hline 3 & 7765 (54.8) & 1167 (57.3) & & 1215 (59.7) & 1167 (57.3) & \\
\hline 4 & $772(5.5)$ & $160(7.9)$ & & $151(7.4)$ & $160(7.9)$ & \\
\hline 5 & $2(0.0)$ & $0(0.0)$ & & $0(0.0)$ & $0(0.0)$ & \\
\hline BMI $\left(\mathrm{kg} / \mathrm{m}^{2}\right)$ & $31.2 \pm 9.1$ & $30.3 \pm 9.0$ & $<0.001$ & 30.2 & 30.4 & 0.021 \\
\hline Caucasian & $9589(67.7)$ & $1295(63.6)$ & $<0.001$ & $1305(64.1)$ & $1295(63.6)$ & 0.026 \\
\hline African-American & $1440(10.2)$ & 169 (8.3) & & $183(9.0)$ & $169(8.3)$ & \\
\hline Other & $673(4.8)$ & $87(4.3)$ & & $97(4.8)$ & $87(4.3)$ & \\
\hline Unknown & 2455 (17.3) & $484(23.8)$ & & $450(22.1)$ & $484(23.8)$ & \\
\hline \multicolumn{7}{|l|}{ Hispanic } \\
\hline Yes & 2103 (14.9) & 451 (22.2) & $<0.001$ & $394(19.4)$ & $451(22.2)$ & 0.045 \\
\hline Unknown & $799(5.6)$ & $67(3.3)$ & & $105(5.1)$ & $67(3.3)$ & \\
\hline \multicolumn{7}{|l|}{ Co-morbidities } \\
\hline Parity & $1.7 \pm 1.6$ & $1.7 \pm 1.5$ & 0.595 & $1.7 \pm 1.5$ & $1.7 \pm 1.5$ & 0.005 \\
\hline \multicolumn{7}{|l|}{ Preoperative laboratories } \\
\hline Platelet count (x109/L) & $287 \pm 124$ & $274 \pm 152$ & 0.04 & $277 \pm 126$ & $274 \pm 152$ & 0.017 \\
\hline Hematocrit (\%) & $34.4 \pm 19.4$ & $33.3 \pm 22.7$ & $<0.001$ & $33.5 \pm 22.3$ & $33.3 \pm 22.4$ & 0.008 \\
\hline History of abdominal/pelvic surgery & $8187(57.8)$ & $1198(58.9)$ & 0.374 & $1178(57.9)$ & $1198(58.9)$ & 0.018 \\
\hline Lymphadenectomy (\% yes) & $9176(64.8)$ & $1266(62.2)$ & 0.022 & $1246(61.2)$ & $1266(62.2)$ & 0.02 \\
\hline \multicolumn{7}{|l|}{ Cancer type } \\
\hline Cervical & $1264(8.9)$ & $115(5.7)$ & $<0.001$ & $117(5.7)$ & $115(5.7)$ & 0.004 \\
\hline Ovarian & $5725(40.4)$ & $1007(49.5)$ & $<0.001$ & $990(48.6)$ & $1007(49.5)$ & 0.014 \\
\hline Uterine & $5885(41.6)$ & $688(33.8)$ & $<0.001$ & $675(33.2)$ & $688(33.8)$ & 0.014 \\
\hline
\end{tabular}

ASA, American Society of Anesthesiology; BMI, body mass index; CHF, congestive heart failure; COPD, chronic obstructive pulmonary disease; SMD, standard mean deviation (no difference if $<0.1$ ), all P-values $>0.05$.

be associated with longer duration of urinary catheterization, a greater number of venous thromboembolism events, Ionger anesthesia time, more frequent vasopressor use, and longer time to ambulation. ${ }^{25-7}$

Given the growing use of multimodal analgesia, including epidurals, this study sought to identify postoperative complications from epidural use in women undergoing hysterectomy for malignancy using a large database. ${ }^{8}$ The primary outcome was the occurrence of 30-day complications reported in the American College of Surgeons' National Surgical Quality Improvement Program dataset, including pulmonary embolism, venous thromboembolism, 
Table 2 Propensity-matched groups - surgical characteristics and 30-day complications

\begin{tabular}{|c|c|c|c|}
\hline Variable (\#, \%), (mean+SD) & $\begin{array}{l}\text { No-epidural } \\
\mathrm{n}=2035\end{array}$ & $\begin{array}{l}\text { Epidural } \\
\mathrm{n}=2035\end{array}$ & P-value \\
\hline Operative time (minutes) & $180 \pm 85$ & $193 \pm 102$ & $<0.001$ \\
\hline Length of hospital stay (days) & $4.79 \pm 4.52$ & $5.69 \pm 5.21$ & $<0.001$ \\
\hline 30-day readmission & $198(9.7)$ & $214(10.5)$ & $<0.001$ \\
\hline 30- day complications (overall) & $1261(62.0)$ & 1545 (75.9) & $<0.001$ \\
\hline Blood transfusion & $463(22.8)$ & 587 (28.9) & $<0.001$ \\
\hline Cerebrovascular accident/stroke & $6(0.3)$ & $11(0.5)$ & 0.225 \\
\hline Myocardial infarction & $12(0.6)$ & $17(0.8)$ & 0.354 \\
\hline Wound disruption & $22(1.1)$ & $40(2.0)$ & 0.022 \\
\hline Surgical site infections & $146(7.2)$ & $205(10.1)$ & 0.001 \\
\hline Deep venous thromboembolism & $24(1.2)$ & $28(1.4)$ & 0.58 \\
\hline Pulmonary embolism & $43(2.1)$ & $38(1.9)$ & 0.570 \\
\hline Sepsis & $109(5.4)$ & $104(5.1)$ & 0.717 \\
\hline Pneumonia & $47(2.3)$ & $45(2.2)$ & 0.827 \\
\hline Urinary tract infection & $66(3.2)$ & $70(3.4)$ & 0.734 \\
\hline Intestinal obstruction & $107(5.3)$ & $116(5.7)$ & 0.543 \\
\hline Prolonged NPO/NGT use & $189(9.3)$ & $250(12.3)$ & 0.002 \\
\hline Prolonged ventilation ( $>48$ hours) & $12(0.6)$ & $13(0.6)$ & 0.844 \\
\hline Unplanned re-intubation & $15(0.7)$ & $21(1.0)$ & 0.065 \\
\hline
\end{tabular}

NGT, nasogastric tube; NPO, nil per os.

pneumonia, and urinary tract infection. Our secondary outcome was to examine the effect of epidural anesthesia on length of stay.

\section{METHODS}

We conducted a retrospective database cohort study using the National Surgical Quality Improvement Program dataset. ${ }^{9}$ This is a large dataset that includes perioperative risk factors and 30-day postoperative outcomes from more than 680 hospitals. Participating hospitals submit surgical cases to the database using strict guidelines to ensure data quality and anonymity of patient information. This dataset was used due to the volume of hysterectomy cases recorded and the ability to capture a large number of epidural cases compared with traditional chart review methods. Disease burden was identified by using pre-operative ascites as a marker for larger disease burden. The dataset is publicly available and includes de-identified data, therefore, this study was considered exempt from review by the Temple University Hospital Institutional Review Board.

Using the 2014-2017 National Surgical Quality Improvement Program dataset procedure-targeted hysterectomy subset, adult patients (18 years or older) who underwent abdominal hysterectomy from January 2014 to December 2017 were identified using common procedure terminology and international classification of diseases codes. Only laparotomy cases were included, and minimally invasive cases (laparoscopy, transvaginal) were excluded due to the small prevalence of epidural cases in this cohort. All patients included in the study were classified as a "gynecologic cancer case" as defined by having a diagnosis of cervical, ovarian, and/ or uterine cancer. All patients received general anesthesia. Epidural analgesia and no-epidural cohorts were defined by the variable "additional anesthetic technique." If patients were noted to have "epidural anesthesia" they were included in the epidural cohort and those receiving other adjuvant techniques (regional blocks or spinal anesthesia) were excluded.

Baseline characteristics between the unmatched cohorts were compared. A multiple logistic regression model was used to calculate a propensity score to control for patient demographics, co-morbidities, oncologic characteristics (type of cancer [ovary, cervical, uterine], ascites). All baseline characteristics were used in the propensity calculation. Patients who received epidural analgesia were matched in a 1:1 fashion with a similar group of patients who did not receive epidural analgesia using the calculated propensity score using the nearest neighborhood method. ${ }^{10}$ This method involves matching a "no-epidural case" and an "epidural case" based on similar characteristics quantified by a propensity score. This allows the two groups to have a "balanced" distribution of covariate characteristics in each group. Propensity-matched groups were assessed for balanced distribution by a standard mean deviation $<0.1$ between groups as acceptable level. ${ }^{11}$ Cohorts were compared using chi-squared test for categorical variables and independent $t$-test for continuous variables.

The primary outcome of interest was the 30-day occurrence of a pulmonary embolism, deep-vein thrombosis, pneumonia, and urinary tract infection. These complications were chosen as they can be linked to prolonged immobility and urinary catheterization with epidural use. Additional 30-day postoperative complications included were: blood transfusion, cerebrovascular accident/stroke, myocardial infarction, wound infection, organ space surgical site infection, sepsis/ septic shock, intestinal obstruction, prolonged nil per os/nasogastric tube, prolonged ventilation ( $>48$ hours), reintubation, and readmission 


\section{Original research}

Table 3 Ovarian cancer subset - operative outcomes and 30-day complications

\begin{tabular}{lllr}
\hline Variable (\#, \%), (mean+SD) & $\begin{array}{l}\text { No-epidural } \\
\mathbf{n = 9 9 0}\end{array}$ & $\begin{array}{l}\text { Epidural } \\
\mathbf{n = 1 0 0 7}\end{array}$ & P-value \\
\hline Operative time (minutes) & $187 \pm 87$ & $202 \pm 101$ & $<0.001$ \\
Length of stay (days) & $5.14 \pm 4.39$ & $5.71 \pm 4.28$ & $<0.001$ \\
Readmission (occurrences) & $90(9.1)$ & $100(9.9)$ & 0.001 \\
30-day complications (overall) & $643(64.9)$ & $784(77.9)$ & $<0.001$ \\
Blood transfusion & $257(25.9)$ & $335(32.3)$ & $<0.001$ \\
Cerebrovascular accident/stroke & $2(0.2)$ & $5(0.5)$ & 0.452 \\
Myocardial infarction & $7(0.7)$ & $4(0.4)$ & 0.350 \\
Wound disruption & $4(0.4)$ & $10(1.0)$ & 0.115 \\
Surgical site infection & $50(5.1)$ & $80(7.9)$ & 0.009 \\
Deep venous thromboembolism & $9(0.9)$ & $15(1.5)$ & 0.234 \\
Pulmonary embolism & $20(2.0)$ & $18(1.8)$ & 0.704 \\
Sepsis & $50(5.1)$ & $55(5.5)$ & 0.681 \\
Pneumonia & $26(2.6)$ & $18(1.8)$ & 0.202 \\
Urinary tract infection & $32(3.2)$ & $37(3.7)$ & 0.589 \\
Intestinal obstruction & $61(6.2)$ & $59(5.9)$ & 0.776 \\
Prolonged NPO/NGT use & $113(11.4)$ & $134(13.3)$ & 0.199 \\
Prolonged ventilation (>48hours) & $5(0.5)$ & $4(0.4)$ & 0.751 \\
Unplanned re-intubation & $7(0.7)$ & $10(1.0)$ & 0.164 \\
\hline
\end{tabular}

NGT, nasogastric tube; NPO, nil per os.

event. Secondary outcomes included length of stay. Complications included were not graded.

All statistical analysis were performed using IBM SPSS statistics version 25 (SPSS, Chicago, IL, USA, 2017).

\section{RESULTS}

A total of 106, 667 women who underwent hysterectomy are included in the database and 42138 of these patients had cervical, ovarian, or uterine cancer. Of the patients with cancer, 16192 underwent a hysterectomy via laparotomy and 2035 patients (13.8\%) of them received both general anesthesia and epidural analgesia. Patient characteristics between unmatched groups were not similar on many variables. The 1:1 propensity-matched samples included 2035 patients in both the no-epidural group and epidural group. Matching on patient characteristics was balanced between the epidural and no-epidural groups as demonstrated by standardized mean difference $<0.1$ (Table 1).

Operative time, length of stay, and 30-day complications between propensity-matched epidural analgesia and no-epidural groups are displayed in Table 2. Patients who received epidural had statistically significantly $(P<0.001)$ longer operative times (mean $192 \mathrm{~min})$ than those who did not $(181 \mathrm{~min})$. The overall 30 -day complication rate was higher in the epidural group compared with the no-epidural group $(75.9 \%$ vs $62.0 \%, P<0.01$, respectively). Compared with the no-epidural group, the proportion of specific complications in the epidural group included a higher rate of blood transfusion $(28.9 \%$ vs $22.8 \%, P<0.01)$, increased rate of wound disruption $(2.0 \%$ vs $1.1 \%$, $P=<0.05)$, increased rate of surgical site infection $(10.1 \%$ vs $7.2 \%$, $\mathrm{P}<0.01$ ), and increased rate of prolonged nil per os or nasogastric tube use $(12.3 \%$ vs $9.3 \%, P<0.01)$. The epidural group experienced a higher rate of readmissions vs the no-epidural group with $10.5 \%$ vs $9.7 \%(P<0.01)$, respectively. The most common reasons for readmission among the 412 patients who were readmitted included wound space/surgical site infection and sepsis. Other 30-day complications were not significantly different between the groups and there was no difference in 30-day mortality, with 13 deaths in the no-epidural group and 18 in the epidural group ( $P=0.62)$. Overall length of stay was significantly longer in the epidural group as compared with the no-epidural group (mean 5.69 days vs 4.79 days, $\mathrm{P}<0.001$ ).

Given a high prevalence of ovarian cancer patients in the cohort (44.7\%), a subset analysis of this group was performed. Baseline characteristics between propensity-matched groups were similar. Operative characteristics and 30-day complications are displayed in Table 3. In patients receiving epidural analgesia, operative time was longer (202 vs $187 \mathrm{~min}, \mathrm{P}<0.001$ ) and these patients experienced a longer length of stay with a mean of 5.71 days compared with 5.14 days in the no-epidural group, $P<0.001$. Similar to the overall group, the ovarian subset demonstrated a higher overall 30 -day complication rate in the epidural group, with $77.9 \%$ experiencing a complication compared with $64.9 \%$ in the no-epidural group $(\mathrm{P}<0.001)$. However, when each complication was analyzed individually, the only difference was a higher blood transfusion rate in the epidural group ( $32.3 \%$ vs $25.9 \%, \mathrm{P}<0.001)$.

\section{DISCUSSION}

Using a large, nationwide database we report an elevated overall 30-day postoperative complication rate in those who received epidural analgesia at time of hysterectomy. We hypothesized that 
complications such as pulmonary embolisms, venous thromboembolisms, pneumonia, and urinary tract infections may be more common in those receiving epidural analgesia, as these can be linked to immobility, but did not see these differences. Instead, in patients who received an epidural we found a higher rate of blood transfusions, wound disruption, surgical site infection, and prolonged nil per os or nasogastric tube use and 1-day longer length of stay.

The use of epidural analgesia in benign gynecologic cases has been widely studied with numerous studies citing improved postoperative pain and faster return to normal bowel function compared with traditional patient controlled analgesia or oral opioid. ${ }^{12-14}$ In gynecologic oncology patients, the use of epidural is not as well studied. Published studies are smaller in size and a majority are retrospective. Furthermore, these studies have not shown consistent results with respect to complications and pain control.

There are conflicting findings over the complications associated with epidural use in the gynecologic oncology population. One of the first studies reported in the gynecologic oncology population used a cohort of 107 patients who received an epidural compared with 98 patients who received patient-controlled analgesia after laparotomy. They reported longer anesthesia time, more use of intraoperative vasopressors, increased intensive care admissions, increased blood transfusion occurrences, and increased time to ambulation among those receiving an epidural. ${ }^{5}$ In a larger study of 237 patients undergoing laparotomy for gynecologic cancer, Courtney-Brooks et al reported improved pain control but found a longer duration of urinary catheterization and a higher incidence of venous thromboembolism. ${ }^{6}$ The study was limited due to the retrospective nature and small complication numbers (five cases in the epidural group and three cases in the no-epidural group). Another retrospective study in 97 patients with gynecologic malignancy compared patient-controlled analgesia to postoperative epidural and found less nausea with epidural, but noted a three-fold increase in urinary tract infections and prolonged urinary catheterization. ${ }^{7}$ While small in size and mostly retrospective, these studies raise a question over the complications associated with epidural use.

Complications were also reported in prospective trials, but the number and size of those trials are limited. A prospective trial in patients undergoing laparotomy for endometrial cancer reported more opioid analgesic use, higher rates of postoperative complications in those who received an epidural $86 \%$ in the epidural group and $66 \%$ in the no-epidural group, $\mathrm{P}<0.01$ ), and a longer length of stay. The complications were not classified as serious or contributing to morbidity/mortality, and included infection and broad categories such as gastrointestinal, neurological, and musculoskeletal complications, but no details were provided. ${ }^{15}{ }^{16}$ Two small, randomized controlled trials reported improved pain control with epidural analgesia and no difference in nausea/emesis, or postoperative ileus. ${ }^{317}$ The first trial included 67 patients undergoing laparotomy for malignancy and the authors noted no difference in complications but reported worse pruritus and increased urinary retention among patients receiving epidural compared with patients receiving patient-controlled analgesia. ${ }^{17}$ The second study, which included 45 patients in the epidural group, noted no difference in rates of pruritus, nausea, or ileus, but reported higher paresthesia and motor weakness in patients who received epidural analgesia compared with patient-controlled intravenous analgesia. ${ }^{3}$
Our study identified higher blood transfusion rate, higher rate of wound complications, and longer duration of nil per os or nasogastric tube use in the epidural group. Although this latter difference was only 3 percentage points higher in the epidural group compared with the no-epidural patients. The time period of our study preceded the publication of enhanced recovery after surgery (ERAS) guidelines for gynecology. The ERAS guidelines encourage early resumption of oral intake and early ambulation. ${ }^{18}$ These strategies may impact the outcomes we observed. However, early ambulation can be compromised in patients who have an epidural.

A study that specifically looked at the impact that an ERAS protocol had on gastrointestinal function after gynecologic cancer surgery included 99 laparotomy patients who received epidural analgesia. They found that epidural use was associated with worse bowel recovery with a 2.6-fold increased likelihood of postoperative ileus $(P=0.02) .^{19}$

Given these mixed findings, we sought to examine the 30-day postoperative complications associated with the use of epidural analgesia in patients undergoing abdominal hysterectomy for gynecologic malignancy using a large, nationwide database. Our data represents the largest cohort studied in this topic, with 2035 patients receiving epidural analgesia included. We hypothesized that complications linked to immobility such as pulmonary embolism, venous thromboembolism, and urinary tract infections may be more common in the epidural group. Although we did not find an increase in these complications, we did find an elevated overall 30-day complication rate in those who received epidural analgesia compared with those who did not $(75.9 \%$ vs $62.0 \%$, respectively, $\mathrm{P}<0.001)$. There were notable differences between the groups with a higher rate of blood transfusions ( 28.9 vs $22.8 \%, P<0.01)$ and higher rates of wound disruption and surgical site infection in the epidural group compared with the no-epidural group. Patients who received an epidural experienced prolonged nil per os or nasogastric tube use more often than patients in the no-epidural group and on average spent almost 1 day longer (mean of 0.9 days) in the hospital. Although a 1-day prolongation of hospitalization may not seem clinically important, it is associated with significant financial loss to the hospital.

A recent study published the largest single-center cohort of 305 patients receiving an epidural.as part of an ERAS protocol. The authors report decreased opioid use and no significant differences in complications. ${ }^{4}$ Our study and the study by Huepenbecker et al both included a large cohort in the epidural group compared with previous studies, whose cohort of patients receiving epidural analgesia were much smaller. ${ }^{4}$ As the rate of postoperative complications are generally low, it is possible that differences in complication rates may only be appreciated in larger cohorts. There is no clear answer as to whether or not epidural analgesia use for gynecologic malignancy is without risk. Several studies including our own demonstrate that epidural use may be associated with a higher rate of complications and prolonged hospitalization, although it could be argued that the differences in the rates of specific complications may not be clinically significant.

There may be beneficial aspects of postoperative epidural that we are not able to address due to study design. Many studies in the gynecologic oncology population report improved pain but this conclusion is not definitively established. Some studies report that patients receiving epidural analgesia report equivalent pain, and 


\section{Original research}

use more morphine equivalents and adjuvant medication than the no-epidural group, ${ }^{515}$ while other studies report improved pain scores, ${ }^{31720}$ and decreased sedation and opioid-side effects. ${ }^{20}$ One study even commented that patient satisfaction with postoperative pain control was significantly improved in the epidural group compared with the patient controlled analgesia group. ${ }^{17}$ To definitely answer these questions, a prospective randomized trial is needed.

Epidural analgesia is increasingly becoming part of ERAS protocols in gynecologic oncology. ${ }^{19}$ These protocols employ multimodal analgesia methods to decrease opioid use and its undesirable side effects, which may lead to faster recovery and shorter hospital stay. Surgeons must consider many aspects of surgical planning and patient care when deciding on which modalities to use for postoperative pain control. Individual patient characteristics are important in making the selection. Further investigation into surgical complexity and utility of epidural with respect to complications should be performed.

On a systems level we must consider the cost that a procedure can have on the patient as well as the healthcare system. In our epidural group, we report higher rates of complications and note a mean 0.9 day longer length of hospital stay. Longer length of stay has also been reported by others. ${ }^{515}$ One study examining American College of Surgeon's data reported that, on average, postsurgical hospitalizations with complications cost $48 \%$ more than uncomplicated surgical hospitalizations. ${ }^{21}$ Our study identified a higher rate of complications in the epidural group, surgical-site infection being one of them. Surgical-site infections is one of the higher cost complications identified by the American College of Surgeons. ${ }^{22}$

While we present the largest cohort of epidural use after hysterectomy for gynecologic cancer, we acknowledge that our findings are limited due to the challenges associated with database studies. The observed increased in 30-day complication rate in the epidural group could be due to the use of epidural analgesia in presumably more complex surgical cases. The epidural group did have a slightly longer operative time than the no-epidural group. However, the percentage of patients undergoing lymphadenectomy was approximately $62 \%$ in the matched comparison groups. Due to the database design we are unable to comment on pain improvement, use of other pain control modalities, and specifics of epidural duration/logistics. Our study reported $13.8 \%$ epidural use, possibly related to NSQIP not consistently capturing these data vs a small prevalence of epidural use in this patient population. Furthermore, ERAS guidelines for gynecology were more widely promoted after the time interval included in our study. The increased complication rate we observed may have diminished if the perioperative care had followed an ERAS protocol. Given the latency in publication of available databases we did not have access to more recent data.

\section{CONCLUSION}

Epidural analgesia is associated with a higher 30-day complication rate and longer hospitalization: however, these complications were not fatal. The benefits of epidural analgesia in patients undergoing hysterectomy for a gynecologic malignancy need to be weighed against the possible complications and the impact a longer hospitalization may have on the patient and healthcare system. A sufficiently powered prospective randomized trial is warranted to further explore this topic.

Twitter Sarah A Ackroyd @drpengu1n

Contributors SAA conceived the subject and design of the study, acquired the dataset, performed the statistical analysis, interpreted the data, and produced the primary draft of the manuscript. EH and KH provided assistance with study design, critique for academic content, analysis and interpretation of the data, and thorough review and revision of the manuscript. MER, CC, GM-S, and SR provided critique for academic content, interpretation of the data, and thorough review and revision of the manuscript. All authors agree with the manuscript results and conclusions.

Funding The authors have not declared a specific grant for this research from any funding agency in the public, commercial, or not-for-profit sectors.

Competing interests None declared.

Patient consent for publication Not required.

Provenance and peer review Not commissioned; externally peer reviewed.

Data availability statement Data may be obtained from a third party and are not publicly available. All data relevant to the study are included in the article or uploaded as supplementary information. NSQIP database- data available as permissible from the American College of Surgeons.

\section{ORCID iD}

Sarah A Ackroyd http://orcid.org/0000-0001-9070-616X

\section{REFERENCES}

1 Jørgensen $\mathrm{H}$, Wetterslev J, Møiniche S, et al. Epidural local anaesthetics versus opioid-based analgesic regimens on postoperative gastrointestinal paralysis, PONV and pain after abdominal surgery. Cochrane Database Syst Rev 2000;4:CD001893.

2 Chinachoti T, Niruthisard S, Tuntisirin O, et al. A double-blind, randomized study comparing postoperative pain management using epidural ropivacaine with intravenous ketorolac or intravenous ketorolac alone following transabdominal hysterectomy. J Med Assoc Thai 2002;85(Suppl 3):S837-47.

3 Moslemi F, Rasooli S, Baybordi A, et al. A comparison of patient controlled epidural analgesia with intravenous patient controlled analgesia for postoperative pain management after major gynecologic oncologic surgeries: a randomized controlled clinical trial. Anesth Pain Med 2015;5:e29540.

4 Huepenbecker SP, Cusworth SE, Kuroki LM, et al. Continuous epidural infusion in gynecologic oncology patients undergoing exploratory laparotomy: the new standard for decreased postoperative pain and opioid use. Gynecol Oncol 2019;153:356-61.

5 Chen L-M, Weinberg VK, Chen C, et al. Perioperative outcomes comparing patient controlled epidural versus intravenous analgesia in gynecologic oncology surgery. Gynecol Oncol 2009;115:357-61.

6 Courtney-Brooks M, Tanner Kurtz KC, Pelkofski EB, et al. Continuous epidural infusion anesthesia and analgesia in gynecologic oncology patients: less pain, more gain? Gynecol Oncol 2015;136:77-81.

7 Elit LM, Thomas H, Trim K, et al. Evaluation of postoperative pain control for women undergoing surgery for gynaecologic malignancies. J Obstet Gynaecol Can 2004;26:1051-8.

8 Nelson G, Kalogera E, Dowdy SC. Enhanced recovery pathways in gynecologic oncology. Gynecol Oncol 2014;135:586-94.

9 American College of Surgeons. User guide for the 2016 ACS NSQIP participant use data file (PUF). Chicago: American College of Surgeons, 2017.

10 Baser $\mathrm{O}$. Too much ado about propensity score models? Comparing methods of propensity score matching. Value Health 2006;9:377-85.

11 Zhang Z, Kim HJ, Lonjon G, et al. Balance diagnostics after propensity score matching. Ann Transl Med 2019;7.

12 Jørgensen H, Fomsgaard JS, Dirks J, et al. Effect of peri- and postoperative epidural anaesthesia on pain and gastrointestinal function after abdominal hysterectomy. $\mathrm{Br} J$ Anaesth 2001;87:577-83.

13 Jørgensen H, Fomsgaard JS, Dirks J, et al. Effect of epidural bupivacaine vs combined epidural bupivacaine and morphine on gastrointestinal function and pain after major gynaecological surgery. Br J Anaesth 2001;87:727-32.

14 Jørgensen H, Fomsgaard JS, Dirks J, et al. Effect of continuous epidural $0.2 \%$ ropivacaine vs $0.2 \%$ bupivacaine on postoperative 
pain, motor block and gastrointestinal function after abdominal hysterectomy. Br J Anaesth 2000;84:144-50.

15 Belavy D, Janda M, Baker J, et al. Epidural analgesia is associated with an increased incidence of postoperative complications in patients requiring an abdominal hysterectomy for early stage endometrial cancer. Gynecol Oncol 2013;131:423-9.

16 Baker J, Janda M, Belavy D, et al. Differences in epidural and analgesic use in patients with apparent stage I endometrial cancer treated by open versus laparoscopic surgery: results from the randomised lace trial. Minim Invasive Surg 2013;2013:764329.

17 Ferguson SE, Malhotra T, Seshan VE, et al. A prospective randomized trial comparing patient-controlled epidural analgesia to patient-controlled intravenous analgesia on postoperative pain control and recovery after major open gynecologic cancer surgery. Gynecol Oncol 2009;114:111-6.

18 Nelson G, Bakkum-Gamez J, Kalogera E, et al. Guidelines for perioperative care in gynecologic/oncology: enhanced recovery after surgery (ERAS) society recommendations - 2019 update. Int $J$ Gynecol Cancer 2019;29:651-68.

19 Boitano TKL, Smith HJ, Rushton T, et al. Impact of enhanced recovery after surgery (ERAS) protocol on gastrointestinal function in gynecologic oncology patients undergoing laparotomy. Gynecol Oncol 2018;151:282-6.

20 Rivard C, Dickson EL, Vogel RI, et al. The effect of anesthesia choice on post-operative outcomes in women undergoing exploratory laparotomy for a suspected gynecologic malignancy. Gynecol Oncol 2014:133:278-82.

21 Dimick JB, Weeks WB, Karia RJ, et al. Who pays for poor surgical quality? Building a business case for quality improvement. J Am Coll Surg 2006;202:933-7.

22 American College of Surgeons. Business case for ACS NSQIP. Chicago, IL: American College of Surgeons, 2020. https://www.facs. org/quality-programs/acs-nsqip/joinnow/businesscase\#3 